\title{
Germanica
}

\section{Norbert Waszek (Hrsg.), G. W. F. Hegel und Hermann Cohen. Wege zur Versöhnung}

\section{Andrée Lerousseau}

\section{OpenEdition}

\section{Journals}

Édition électronique

URL : https://journals.openedition.org/germanica/8238

DOI : 10.4000/germanica.8238

ISSN : 2107-0784

Éditeur

Université de Lille

Édition imprimée

Date de publication : 1 décembre 2019

Pagination : 189-192

ISBN : 978-2-913857-44-5

ISSN : 0984-2632

Référence électronique

Andrée Lerousseau, « Norbert Waszek (Hrsg.), G. W. F. Hegel und Hermann Cohen. Wege zur

Versöhnung », Germanica [En ligne], 65 | 2019, mis en ligne le 01 janvier 2021, consulté le 08 janvier 2022. URL : http://journals.openedition.org/germanica/8238 ; DOI : https://doi.org/10.4000/ germanica.8238

Ce document a été généré automatiquement le 8 janvier 2022

(C) Tous droits réservés 


\title{
Norbert Waszek (Hrsg.), G. W. F. Hegel und Hermann Cohen. Wege zur Versöhnung
}

\author{
Andrée Lerousseau
}

\section{RÉFÉRENCE}

Festschrift für Myriam Bienenstock, Freiburg/ München, Verlag Karl Alber, 270 p.

1 Fruit de parentés et d'affinités intellectuelles, d'amitiés, de compagnonnages et d'émulation réciproque, cette publication est un hommage aux travaux de Myriam Bienenstock dont l'œuvre, ainsi que le rappelle Dana Hollander, constitue une contribution unique, d'un niveau rarement atteint par d'autres penseurs, aux études consacrées à la philosophie européenne moderne et à sa réception dans la pensée juive moderne ${ }^{1}$. D'où une première série de contributions portant sur l'idéalisme allemand et en particulier sur Hegel, suivie de trois études autour de Hermann Cohen et du néokantisme.

La parole est donnée en premier lieu à Bernard Bourgeois qui expose en une synthèse magistrale les débuts de l'histoire universelle et le sens que revêt celle-ci chez Hegel, conviant le lecteur à cheminer d'Est en Ouest, de «l'enfance de l'histoire » en Chine, puis en Inde, à la « coupure anthropologique » marquée par la Perse libérant « l'histoire et la politique [...] pour la liberté dont l'Europe sera la réalisation achevée ». Dans le second chapitre, Christophe Bouton analyse en quoi le concept hégélien d'ethnicité, intrinsèquement lié à la notion d'historicité, serait mieux à même de penser et de définir les devoirs et d'insuffler un comportement moral que l'impératif catégorique atemporel et par trop formel de Kant. L'auteur s'inscrit en outre en faux contre Axel Honneth qui envisageait un recours à la morale kantienne comme «morale de secours en situation de crise » : pour Hegel, allègue Christophe Bouton, c'est bien plutôt la morale socratique qui peut servir « de relève à une ethnicité défaillante » (défaillances dont il fournit des illustrations à travers la lecture de plusieurs des plus beaux passages 
de la Phénoménologie). L'étude de Gerhard Kurz portant sur le «besoin » trouve son impulsion dans le numéro de la Revue Germanique internationale parue en 2002 sous la direction de Myriam Bienenstock consacré à la notion de «Trieb » (Trieb : tendance, instinct, pulsion), qui lui permet de dresser un «état des lieux » dans un rappel du sens que revêt dans ce champ conceptuel et chez différents penseurs la notion de «besoin » à une époque où se produit un « tournant décisif » (M. Bienenstock) et où ces différents concepts acquièrent une dimension anthropologique. Un exemple est fourni par l'anthropologisation à laquelle procède Hölderlin du système des pulsions (Triebstruktur) développé par Fichte. Habité par une double aspiration, à la fois à l'absolu et à la limitation, l'homme éprouve le besoin d'une philosophie de la réconciliation esquissée dans le fragment en prose intitulé Hyperions Jugend. La contribution de Claudia Melika est la reprise d'une conférence qui avait attiré l'attention de Myriam Bienenstock voyant là le prolongement de ses propres réflexions autour de Hegel et d'Aristote entamées en 1989. L'auteure analyse la reprise par Hegel, considéré par Alain comme "l'Aristote des temps modernes", de plusieurs thèses développées par le Stagirite, ainsi par exemple celle de la notion d'entéléchie, introduisant un mouvement de circularité, et démontre la façon dont Hegel effectue une relecture de la notion de «finalité interne » de Kant, « simple maxime du jugement réfléchissant » sans effectivité, au prisme du concept aristotélicien de "vie » afin de parvenir à la "vérité objective " réconciliant la réalité et le concept. Wolfdietrich Schmied-Kowarzik s'interroge quant à lui sur la divergence - évoquée par Schelling dans une lettre à Hegel datée de novembre 1807 - ayant entraîné la rupture entre les deux amis ( ... versöhnen läßt sich freilich Alles, Eines ausgenommen ... »). Si, en quête de "l'unité absolue de la raison et de la réalité », l'un comme l'autre ont été amenés à congédier l'idéalisme subjectif de Fichte, leur désaccord réside dans leur façon d'aborder l'absolu : là où Hegel développe une phénoménologie de l'esprit, Schelling tel est le reproche de Hegel - en resterait au stade intermédiaire de la contemplation de l'absolu dans l'œuvre d'art, au sentiment de l'absolu et non à sa conceptualisation ("phénoménologie » versus "odyssée » pour reprendre la distinction établie par Philippe Grosos dans un bel article paru en 2016). Dans le prolongement d'un séminaire organisé avec Myriam Bienenstock, Ludwig Siep développe une réflexion sur «la dimension religieuse dans la pensée de Hegel ». Acquiesçant à la thèse de Fackenheim selon lequel la philosophie de l'absolu de Hegel revêt bien une dimension religieuse, l'auteur juge toutefois que le prix à payer pour la réconciliation - notion en soi religieuse - est trop lourd. Si Fackenheim juge que la «synthèse » réalisée par Hegel n'est plus valable pour le présent, Ludwig Siep considère quant à lui que Hegel n'aurait jamais atteint son but, sa philosophie «religieuse» n'en demeurant pas moins stimulante pour penser une "philosophie non-religieuse ». Norbert Waszek relève, à travers la lecture que fait Heine des "figures de proue» de l'Aufklärung que sont Lessing et Mendelssohn, la double tâche qui s'impose à lui, désireux à la fois de s'inscrire dans une continuité (poursuivre à l'instar des membres du Kulturverein la critique de la tradition entamée par l'Aufklärung), et de dépasser un déisme appartenant au passé : il convient pour le présent de se hisser au panthéisme, seul garant d'harmonie, en renouant avec Spinoza. Si Myriam Bienenstock s'attachait dans un article majeur paru en 2001 à retrouver l'esprit objectif hégélien «tel qu'en luimême ", dégagé des strates de l'interprétation, Jean-François Goubet concentre pour sa part son attention sur deux interprétations, sur la « reconfiguration » de cette notion hégélienne chez les herbartiens Theodor Waitz et Moritz Lazarus, le premier dénonçant 
un certain élitisme chez Hegel et une incapacité, pour tout hégélien, à développer une véritable pédagogie et une psychologie de l'individu, le second mettant l'accent sur le collectif (la psychologie des peuples et une linguistique nouvelle) et tendant à confondre l'esprit objectif avec «l'esprit objectivé» (reproche émis par Myriam Bienenstock à l'égard de Dilthey). Il n'en demeure pas moins que l'héritage conjugué de Hegel et de Herbart, auxquels il convient d'ajouter Durkheim, est à même d'alimenter la réflexion autour des processus de socialisation.

Viennent ensuite trois contributions relatives à l'école néokantienne de Marbourg. Pierfrancesco Fiorato se penche sur la lecture que fait Cohen de la philosophie de l'histoire de Kant. Si Kant est bien le précurseur d'une philosophie de l'histoire, il ne parvient pas pour autant à résoudre le " problème de l'histoire ", en ce qu'il ne fournit pas «les moyens méthodiques adaptés » à la réalisation concrète de l'histoire, en d'autres termes: il n'accède pas à la "maturité historique ». La tâche que s'assigne Cohen est donc de réfléchir à ce qui assure le lien entre une conception téléologicomorale un peu trop naïve et optimiste et les « conditions économiques matérielles », au moyen - à ses yeux le droit - qui permet à l'individu de devenir "sujet de l'histoire ", sans oublier la prise en considération du «moment irrationnel» qui trouve son expression dans les crises ou les révolutions. Helmut Holzhey prête quant à lui l'oreille au "grondement » sous-jacent produit «par les questions métaphysiques" dans les considérations développées par le néokantisme autour de l'âme ou de Dieu. Quels que soient les recours, les hypothèses, les déplacements dans l'appréhension de ces idées, il demeurerait comme un écho de la métaphysique, une « rechute dans une métaphysique antérieure à Kant ». L'exposé de Dana Hollander enfin apporte un éclairage nouveau ou complémentaire sur le rapport conflictuel qu'entretient Cohen à Spinoza qui n'a eu de cesse de stigmatiser le particularisme et l'exclusivisme juifs: l'auteure démontre comment, paradoxalement, la critique que fait Cohen de la "politisation du judaïsme » chez Spinoza le conduit non pas à une "dépolitisation », mais à une "réévaluation » de la dimension politique du judaïsme dans le sens d'un messianisme « au service d'une politique internationale universaliste ».

Les deux dernières contributions occupent une place un peu à part. Dominique Bourel consacre son exposé à l'un des précurseurs de la rencontre et du débat entre la philosophie allemande moderne et la pensée juive moderne, le philosophe shmuel Hugo Bergmann qui, après avoir effectué son alya en 1920, deviendra le premier directeur de la Bibliothèque Nationale juive et le premier recteur de l'Université hébraïque de Jérusalem (1935-1938) où il enseigne la philosophie. "Passeur de la pensée européenne ", il participe à l'élaboration d'un lexique philosophique en hébreu et traduit entre autres, en collaboration avec Nathan Rotenstreich, les trois critiques de Kant. L'article s'attarde également sur l'engagement de ce sioniste de la première heure qui tentera d'œuvrer, à l'instar de Buber, au rapprochement entre Juifs et Arabes. Entamé en Allemagne, le voyage se poursuit donc jusqu'à cette "Terre promise » dont la contribution de Guy S. Stroumsa conte les avatars, énumère les métamorphoses et les déplacements du sens et des interprétations faites par les différents descendants d'Abraham, avant de proposer, dans la lignée de Hegel, comme solution aux conflits nés autour de cette notion, une sorte de Aufhebung, d'abolition du discours religieux et eschatologique dans une "mémoire culturelle».

À travers ces études qui témoignent de la stimulation exercée par l'œuvre de Myriam Bienenstock et de la fécondité des échanges, le lecteur effectue une formidable plongée 
dans ce que nous serions tentée de nommer, en référence à Hannah Arendt, « la vie de l'esprit ».

\section{NOTES}

1. . - Voir à ce propos notre recension de son dernier essai, Cohen und Rosenzweig. Ihre Auseinandersetzung mit dem deutschen Idealismus, dans le précédent numéro de Germanica (64/2019). 